\title{
Axiomatization of fuzzy attribute logic over complete residuated lattices
}

\author{
Radim Bělohlávek Vilém Vychodil \\ Dept. Computer Science, Palacký University, Tomkova 40, CZ-779 00, Olomouc, Czech Republic \\ Email: \{radim.belohlavek, vilem.vychodil\}@upol.cz
}

\begin{abstract}
The paper deals with fuzzy attribute logic (FAL) and shows its completeness over all complete residuated lattices. FAL is a calculus for reasoning with if-then rules describing particular attribute dependencies in objectattribute data. Completeness is proved in two versions: classical-style completeness and graded-style completeness.
\end{abstract}

Keywords: completeness, residuated lattice, fuzzy logic, fuzzy attribute, graded completeness, if-then rule

\section{Introduction}

It has been well recognized that extracting of patterns from data and further analysis of the obtained patterns is an important task which may help discover hidden dependencies in data. If-then rules are perhaps the most widely used patterns to be extracted from object-attribute data. The increasing interest in fuzzy logic in both the narrow and wide sense calls for methods for reasoning with if-then rules in fuzzy setting. In our previous papers $[2,3,4,5,6]$ we introduced fuzzy attribute logic which was originally motivated by problems in data analysis (generating of non-redundant bases from given object-attribute data with fuzzy attributes [2]). Since then, fuzzy attribute logic has evolved into a full-fledged logical calculus and proved to be useful in various areas (formal concept analysis, database systems, etc., see [6] for an overview of the recent results). Fuzzy attribute logic (FAL) deals with rules, called fuzzy attribute implications, of the form "if $A$ then $B$ " where $A$ and $B$ are collections of attributes, with the meaning: if an object has all the attributes of $A$ then it has also all attributes of $B$. In $[3,5]$ we showed several axiomatizations of FAL and proved its completeness over finite residuated lattices with hedges. This seems to be enough when considering the applications. For instance, finite object-attribute tables from which the if-then rules are generated can contain only finitely many truth degrees which can be captured in a finite structure. On the other hand, axiomatization of FAL over infinite structures of truth degrees seem to be an interesting problem. The present paper shows that
FAL is complete for any complete residuated lattices with hedges. This class of structures of truth degrees include, among other structures, residuated lattices on the real unit interval given by left-continuous t-norms. We prove two types of completeness theorems: classical-style completeness (provability is a bivalent notion) and graded-style completeness (provability is a matter of degree).

\section{Preliminaries}

Fuzzy attribute logic is developed over complete residuated lattices with truth-stressing hedges (shortly, hedges). A complete residuated lattice with a hedge, which is our basic structure of truth degrees, is an algebra $\mathbf{L}=$ $\left\langle L, \wedge, \vee, \otimes, \rightarrow,{ }^{*}, 0,1\right\rangle$ such that $\langle L, \wedge, \vee, 0,1\rangle$ is a complete lattice with 0 and 1 being the least and greatest element of $L$, respectively; $\langle L, \otimes, 1\rangle$ is a commutative monoid (i.e. $\otimes$ is commutative, associative, and $a \otimes 1=$ $1 \otimes a=a$ for each $a \in L) ; \otimes$ and $\rightarrow$ satisfy so-called adjointness property: $a \otimes b \leq c$ iff $a \leq b \rightarrow c$; for each $a, b, c \in L$; hedge * satisfies (i) $1^{*}=1$, (ii) $a^{*} \leq a$, (iii) $(a \rightarrow b)^{*} \leq a^{*} \rightarrow b^{*}$, (iv) $a^{* *}=a^{*}$, for all $a, b \in L$. Elements $a$ of $L$ are called truth degrees. $\otimes$ and $\rightarrow$ are (truth functions of) "fuzzy conjunction" and "fuzzy implication". Hedge * is a (truth function of) logical connective "very true", see $[8,9]$. Properties (i)-(iv) have natural interpretations, e.g. (iii) can be read: "if $a \rightarrow b$ is very true and if $a$ is very true, then $b$ is very true", etc.

A common choice of $\mathbf{L}$ is a structure with $L=[0,1]$ (unit interval), $\wedge$ and $\vee$ being minimum and maximum, $\otimes$ being a left-continuous t-norm with the corresponding $\rightarrow$. Three most important pairs of adjoint operations on the unit interval are: Łukasiewicz $(a \otimes b=\max (a+b-1,0)$, $a \rightarrow b=\min (1-a+b, 1))$, Gödel: $(a \otimes b=\min (a, b)$, $a \rightarrow b=1$ if $a \leq b, a \rightarrow b=b$ else), Goguen (product): $\left(a \otimes b=a \cdot b, a \rightarrow b=1\right.$ if $a \leq b, a \rightarrow b=\frac{b}{a}$ else). Complete residuated lattices include also finite structures of truth degrees. For instance, we can take a finite subset $L \subseteq[0,1]$ that is closed under Łukasiewicz or Gödel operations. If we take $L=\{0,1\}$, we obtain this way the two-element Boolean algebra (structure of truth degrees of classical logic). Two boundary cases of hedges are (i) identity, i.e. $a^{*}=a(a \in L)$; (ii) globalization [11]: $a^{*}=1$ if $a=1, a^{*}=0$ else. 
Given $\mathbf{L}$ which serves as a structure of truth degrees, we define usual notions: an L-set (fuzzy set) $A$ in universe $U$ is a mapping $A: U \rightarrow L, A(u)$ being interpreted as "the degree to which $u$ belongs to $A$ ". Let $\mathbf{L}^{U}$ denote the collection of all $\mathbf{L}$-sets in $U$. The operations with $\mathbf{L}$-sets are defined componentwise. For instance, intersection of $\mathbf{L}$-sets $A, B \in \mathbf{L}^{U}$ is an $\mathbf{L}$-set $A \cap B$ in $U$ such that $(A \cap B)(u)=$ $A(u) \wedge B(u)$ for each $u \in U$, etc. For $a \in L$ and $A \in \mathbf{L}^{U}$, we define L-sets $a \otimes A$ ( $a$-multiple of $A)$ and $a \rightarrow A$ ( $a$-shift of $A)$ by $(a \otimes A)(u)=a \otimes A(u),(a \rightarrow A)(u)=a \rightarrow A(u)$ $(u \in U)$. Given $A, B \in \mathbf{L}^{U}$, we define a subsethood degree $S(A, B)=\bigwedge_{u \in U}(A(u) \rightarrow B(u))$, which generalizes the classical subsethood relation $\subseteq$. Described verbally, $S(A, B)$ represents the degree to which $A$ is a subset of $B$. In particular, we write $A \subseteq B$ iff $S(A, B)=1$. We have $A \subseteq B$ iff, for each $u \in U, A(u) \leq B(u)$. In the following we use well-known properties of residuated lattices and fuzzy structures which can be found in monographs $[1,8]$.

\section{Fuzzy attribute implications}

\subsection{Validity and semantic entailment}

In this section we recall basic notions of fuzzy attribute logic (FAL). More details can be found in $[2,4,6]$.

In what follows, we let $Y$ be a finite set of attributes, each $y \in Y$ will be called an attribute. Fuzzy attribute implication (over attributes $Y$ ) is an expression $A \Rightarrow B$, where $A, B \in \mathbf{L}^{Y}$ ( $A$ and $B$ are fuzzy sets of attributes). Fuzzy attribute implications (FAIs) are the formulas of fuzzy attribute logic. In order to consider validity (truth) of FAIs, we introduce a semantic component in which we evaluate FAIs and their formal interpretation. The intuitive meaning we wish to give to $A \Rightarrow B$ is: "if it is (very) true that an object has all attributes from $A$, then it has also all attributes from $B$ '. Formally, for an $\mathbf{L}$-set $M \in \mathbf{L}^{Y}$ of attributes, we define a degree $\|A \Rightarrow B\|_{M} \in L$ to which $A \Rightarrow B$ is true in $M$ by

$$
\|A \Rightarrow B\|_{M}=S(A, M)^{*} \rightarrow S(B, M)
$$

where $S(\cdots)$ denote subsethood degrees, see Section 2 . The degree $\|A \Rightarrow B\|_{M}$ can be understood as follows: if $M$ (semantic component) represents presence of attributes of some object, i.e. $M(y)$ is truth degree to which "the object has the attribute $y \in Y$ ", then $\|A \Rightarrow B\|_{M}$ is the truth degree to which "if the object has all attributes from $A$, then it has all attributes from $B$ ", which corresponds to the desired interpretation of $A \Rightarrow B$. Note also that the hedge ${ }^{*}$ servers as a modifier of interpretation of $A \Rightarrow B$, see $[2,4,6]$ for details.

Let $T$ be a set of fuzzy attribute implications. $M \in \mathbf{L}^{Y}$ is called a model of $T$ if $\|A \Rightarrow B\|_{M}=1$ for each $A \Rightarrow B \in$ $T$. The set of all models of $T$ is denoted by $\operatorname{Mod}(T)$. A degree $\|A \Rightarrow B\|_{T} \in L$ to which $A \Rightarrow B$ semantically follows from $T$ is defined by

$$
\|A \Rightarrow B\|_{T}=\bigwedge_{M \in \operatorname{Mod}(T)}\|A \Rightarrow B\|_{M} .
$$

Described verbally, $\|A \Rightarrow B\|_{T}$ is defined as a degree to which " $A \Rightarrow B$ is true in each model of $T$ ". Hence, $\|\cdots\|_{T}$ defined by (2) represents a degree of semantic entailment from $T$. In further sections we will be interested in syntactic characterizations of $\|\cdots\|_{T}$.

\subsection{Provability and syntactic entailment}

This section introduces a deductive system for fuzzy attribute logic and a particular notion of provability which generalize the ones presented in [5]. The generalized notions will allow us to prove various completenes theorems (in Section 4.) over each complete residuated lattice with hedge taken as the structure of truth degrees.

Deductive systems for FAL presented in $[3,5]$ are based on deduction rules of the form "from $\varphi_{1}, \ldots, \varphi_{n}$ infer $\varphi$ ", where $n$ is a nonnegative integer and $\varphi, \varphi_{i}(i \in I)$ are (schemas for) FAIs. Such deduction rules are to be understood as usual: having rule "from $\varphi_{1}, \ldots, \varphi_{n}$ infer $\varphi$ " and FAIs which are of the form of FAIs in the input part (the part preceding "infer") of the rule, the rule allows us to infer (in one step) the corresponding FAI in the output part (the part following "infer") of the rule. Each nullary rule, i.e. rule where $n=0$, is considered as axiom the output part of which can be inferred in one step.

Deductive system of FAL which has been introduced in [5] uses the following rules.

(Ax) infer $A \cup B \Rightarrow A$,

(Cut) from $A \Rightarrow B$ and $B \cup C \Rightarrow D$ infer $A \cup C \Rightarrow D$,

(Mul) from $A \Rightarrow B$ infer $c^{*} \otimes A \Rightarrow c^{*} \otimes B$

for each $A, B, C, D \in \mathbf{L}^{Y}$, and $c \in L$. Notice that $(\mathrm{Ax})$ is a nullary rule (axiom) which says that each $A \cup B \Rightarrow A$ $\left(A, B \in \mathbf{L}^{Y}\right)$ is inferred in one step. The rules are inspired by Armstrong-like axioms, see [10] for a good overview.

A fuzzy attribute implication $A \Rightarrow B$ is called provable from a set $T$ of FAIs using a set $\mathcal{R}$ of deduction rules if there is a sequence $\varphi_{1}, \ldots, \varphi_{n}$ of fuzzy attribute implications such that $\varphi_{n}$ is $A \Rightarrow B$ and for each $\varphi_{i}$ we either have $\varphi_{i} \in T$ or $\varphi_{i}$ is inferred (in one step) from some of the preceding formulas using some deduction rule from $\mathcal{R}$ (i.e., $R$ contains a rule "from $\psi_{1}, \ldots, \psi_{k}$ infer $\varphi_{i}$ " where each of $\psi_{1}, \ldots, \psi_{k}$ is among $\left.\varphi_{1}, \ldots, \varphi_{i-1}\right)$. If $\mathcal{R}$ consists of (Ax)(Mul), we say just " $A \Rightarrow B$ is provable from $T$ " instead of " $A \Rightarrow B$ is provable from $T$ using ..." and denote this fact by $T \vdash A \Rightarrow B$.

A deduction rule "from $\varphi_{1}, \ldots, \varphi_{n}$ infer $\varphi$ " is said to be sound if $\operatorname{Mod}\left(\left\{\varphi_{1}, \ldots, \varphi_{n}\right\}\right) \subseteq \operatorname{Mod}(\{\varphi\})$. A deduction rule "from $\varphi_{1}, \ldots, \varphi_{n}$ infer $\varphi$ " $\left(\varphi_{i}, \varphi\right.$ are FAIs $)$ is said to be derivable (from a set $\mathcal{R}$ of deduction rules) if $\varphi$ is 
provable from $\left\{\varphi_{1}, \ldots, \varphi_{n}\right\}$ (using $\mathcal{R}$ ). The following observation will be used in the sequel.

Lemma 1 (see [5]) The following deduction rules are derivable from $(\mathrm{Ax})$ and $(\mathrm{Cut})$ :

(Add) from $A \Rightarrow B$ and $A \Rightarrow C$ infer $A \Rightarrow B \cup C$,

(Pro) from $A \Rightarrow B \cup C$ infer $A \Rightarrow B$,

(Tra) from $A \Rightarrow B$ and $B \Rightarrow C$ infer $A \Rightarrow C$,

for each $A, B, C, D \in \mathbf{L}^{Y}$.

In $[3,5]$, we showed that given a finite residuated lattice with a hedge, we have that $T \vdash A \Rightarrow B$ iff $\|A \Rightarrow B\|_{T}=$ 1. Described verbally, FAIs which are semantically entailed from $T$ to degree 1 (fully entailed) are exactly the FAIs which are provable from $T$ using (Ax), (Cut), and (Mul). We now extend the deductive system of FAL so that we will be able to prove this claim (and much more) for any complete residuated lattice with a hedge.

The present deductive system will be extended by the following infinitary deduction rule which can have infinitely many FAIs in the input part (part preceding "infer"):

$\left(\operatorname{Add}_{\omega}\right)$ from $A \Rightarrow B_{i}(i \in I)$ infer $A \Rightarrow \bigcup_{i \in I} B_{i}$, where $\left\{A \Rightarrow B_{i} \mid i \in I\right\}$ is an $I$-indexed set of FAIs. Thus, $\left(\operatorname{Add}_{\omega}\right)$ is of the form "from $\varphi_{i}(i \in I)$ infer $\varphi$ ". Rules of this form will be called $\omega$-deduction rules (shortly, $\omega$ rules). Clearly, (Ax), (Cut), and (Mul) can also be seen as $\omega$-rules, where $I$ is finite. Note that infinitary rules are widely used in logic and computer science. For instance, they are used in universal algebra [12] as well as fuzzy logic in narrow sense (e.g., in TT $\forall$, see [8]). From now our, assume that we use a deductive system which consists of (Ax), (Cut), (Mul), and ( $\left.\operatorname{Add}_{\omega}\right)$.

In order to use $\omega$-rules we switch from proofs considered as finite sequences of FAIs to $\omega$-proofs which will be defined as certain labeled infinitely branching rooted (directed) trees with finite depth [7, 12]. Each tree will be denoted by $\mathcal{T}=\langle l, S t\rangle$, where $l$ is a label in the root of $\mathcal{T}$, and $S t$ is a set of subtrees of $\mathcal{T}: S t=\left\{\mathcal{T}_{v} \mid \mathcal{T}_{v}\right.$ is a subtree at $v$, and $v$ is a direct descendant of the root of $\mathcal{T}\}$, see [7] Leaf nodes are thus denoted $\mathcal{T}=\langle l, \emptyset\rangle$.

Given a set $T$ of fuzzy attribute implications, we define an $\omega$-proof from $T$ (using deductive system $\mathcal{R}$ ) as follows: (i) for each $A \Rightarrow B \in T$, tuple $\mathcal{T}=\langle A \Rightarrow B, \emptyset\rangle$ is an $\omega$-proof from $T$ (using $\mathcal{R}$ ); (ii) if $\mathcal{T}_{i}=\left\langle\varphi_{i}, \ldots\right\rangle(i \in I)$ are $\omega$-proofs from $T$ (using $\mathcal{R}$ ) and "from $\varphi_{i}(i \in I)$ infer $\varphi$ " is a rule in $\mathcal{R}$ then $\mathcal{T}=\left\langle\varphi,\left\{\mathcal{T}_{i} \mid i \in I\right\}\right\rangle$ is an $\omega$-proof from $T$ (using $\mathcal{R}$ ). $A \Rightarrow B$ is called $\omega$-provable from $T$ (using $\mathcal{R})$, written $T \vdash_{\omega} A \Rightarrow B\left(T \vdash_{\omega, \mathcal{R}} A \Rightarrow B\right)$, if there is an $\omega$-proof $\mathcal{T}$ from $T$ (using $\mathcal{R}$ ) such that $\mathcal{T}=\langle A \Rightarrow B, \ldots\rangle$ (i.e., if $A \Rightarrow B$ is the label in the root node of $\mathcal{T}$ ). One can introduce notions of soundness and $\omega$-derivability of $\omega$-rules as in the previous setting.
The following assertion shows that, considering only (Ax), (Cut), and (Mul), provability coincides with $\omega$ provability.

Theorem $2 T \vdash A \Rightarrow B$ iff $A \Rightarrow B$ is $\omega$-provable from $T$ using (Ax), (Cut), and (Mul).

Proof. " $\Rightarrow$ ": Let $T \vdash A \Rightarrow B$. Thus, there is a sequence of FAIs $\varphi_{1}, \ldots, \varphi_{n}$ which is a proof of $A \Rightarrow B$ from $T$. By induction, we can check that for each $\varphi_{i}(i=1, \ldots, n)$ there is an $\omega$-proof of $\varphi_{j}$.

" $\Leftarrow "$ : Let $A \Rightarrow B$ is $\omega$-provable from $T$ using (Ax), (Cut), and (Mul). Thus, there is an $\omega$-proof $\mathcal{T}$ using (Ax), (Cut), and (Mul) which is finite. Now, one can construct a sequence of FAIs by depth-first traversing $\mathcal{T}$ and listing all labels in post-order. This sequence is a proof of $A \Rightarrow B$ from $T$ (easy to check).

The following assertion shows that if we use finite structures of truth degrees, $\omega$-provability yields exactly the same as (the usual) provability. In this sense, our extension of the deductive system of FAL is conservative with respect to the previous results.

Theorem 3 Let $\mathbf{L}$ be a finite residuated lattice with hedge. Then, for each set $T$ of FAIs and for each $A \Rightarrow B$,

$$
T \vdash{ }_{\omega} A \Rightarrow B \text { iff } T \vdash A \Rightarrow B \text { iff }\|A \Rightarrow B\|_{T}=1 .
$$

Proof. It suffices to show $T \vdash{ }_{\omega} A \Rightarrow B$ iff $T \vdash A \Rightarrow B$. The rest is a consequence of the completeness of FAL for finite structures of truth degrees, see [3].

" $\Rightarrow$ ": The crucial observation here is that since both $L$ and $Y$ are finite, we can equivalently replace $\left(\operatorname{Add}_{\omega}\right)$ by a collection of rules

$\left(\operatorname{Add}_{n}\right)$ from $A \Rightarrow B_{1}, \ldots, A \Rightarrow B_{n}$ infer $A \Rightarrow B_{1} \cup \cdots \cup B_{n}$, for each $n$ being a nonnegative integer. Each $\left(\operatorname{Add}_{n}\right)$ is derivable from (Ax), (Cut), and (Mul), see Lemma 1. Hence, if $T \vdash_{\omega} A \Rightarrow B$, then there is a finite $\omega$-proof $\mathcal{T}$ of $A \Rightarrow B$ from $T$ which uses only (Ax), (Cut), and (Mul). Now apply Theorem 2 .

$$
\text { " } \Leftarrow \text { ": Follows from Theorem } 2 \text {. }
$$

Remark 4 For general $\mathbf{L},\left(\operatorname{Add}_{\omega}\right)$ is not $\omega$-derivable from (Ax)-(Mul). Indeed, let $L=[0,1]$ and $Y=\{y\}$. It suffices to show that FAI \{\}$\Rightarrow\{y\}$ is not $\omega$-provable from a set $T=\{\{\} \Rightarrow\{a / y\} \mid a<1\}$ using (Ax)-(Mul). By contradiction, let $\mathcal{T}$ be an $\omega$-proof of \{\}$\Rightarrow\{y\}$ from $T$ which uses only rules (Ax), (Cut), and (Mul). By Theorem 2, $T \vdash\{\} \Rightarrow\{y\}$. By a standard argument, there is a finite subset $T^{\prime}$ of $T$ such that $T^{\prime} \vdash\{\} \Rightarrow\{y\}$. Now, for $b=\bigvee\left\{a \in L \mid\{\} \Rightarrow\{a / y\} \in T^{\prime}\right\}$ we have $b<1$. Since $L=[0,1]$, one can take a model $M \in \mathbf{L}^{Y}$ of $T^{\prime}$ such that $b<M(y)<1$. For $M$, we have $\|\{\} \Rightarrow\{y\}\|_{M}=M(y)$. The latter observation yields $\|\{\} \Rightarrow\{y\}\|_{T^{\prime}}<1$. Since (Ax)-(Mul) are sound [5], we obtain $T^{\prime} \forall\{\} \Rightarrow\{y\}$, a contradiction. 


\section{Soundness and completeness}

We now prove completeness of FAL in two versions. First, we show that FAIs which are $\omega$-provable from $T$ are those which semantically follow from $T$ to degree 1 (full truth). Recall that we assume that $\mathbf{L}$ is a complete residuated lattice with a hedge.

Theorem 5 (soundness) $\left(\operatorname{Add}_{\omega}\right)$ is sound. Moreover, for each set $T$ of FAIs and each $A \Rightarrow B$ we have: if $T \vdash_{\omega} A \Rightarrow$ $B$, then $\|A \Rightarrow B\|_{T}=1$.

Proof. Let $T=\left\{A \Rightarrow B_{i} \mid i \in I\right\}$ be a set of FAIs, and let $M \in \operatorname{Mod}(T)$. Then, for each $i \in I,\left\|A \Rightarrow B_{i}\right\|_{M}=$ 1 , which is equivalent to $S(A, M)^{*} \leq S\left(B_{i}, M\right)$. Thus, $S(A, M)^{*} \leq \bigwedge_{i \in I} S\left(B_{i}, M\right)$, which further gives $S(A, M)^{*} \leq$ $S\left(\bigcup_{i \in I} B_{i}, M\right)$, i.e. $\left\|A \Rightarrow \bigcup_{i \in I} B_{i}\right\|_{M}=1$, showing that $\left(\operatorname{Add}_{\omega}\right)$ is sound. The rest can be proved by induction using the fact that (Ax), (Cut), and (Mul) are sound [5].

Lemma 6 Let $T$ be a set of FAIs. If $T \forall_{\omega} A \Rightarrow B$, then there is a model $M \in \operatorname{Mod}(T)$ such that $\|A \Rightarrow B\|_{M} \neq 1$.

Proof. Let $T \nvdash_{\omega} A \Rightarrow B$. Put $M=\bigcup\left\{C \in \mathbf{L}^{Y} \mid T \vdash_{\omega}\right.$ $A \Rightarrow C\}$. We have that $T \vdash_{\omega} A \Rightarrow M$. Indeed, for each $T \vdash{ }_{\omega} A \Rightarrow C$, there is an $\omega$-proof $\mathcal{T}_{C}$ of $A \Rightarrow C$ from $T$. Hence, using $\left(\operatorname{Add}_{\omega}\right), \mathcal{T}=\left\langle A \Rightarrow M,\left\{\mathcal{I}_{C} \mid T \vdash_{\omega} A \Rightarrow C\right\}\right\rangle$ is an $\omega$-proof of $A \Rightarrow M$ from $T$, showing $T \vdash_{\omega} A \Rightarrow M$.

Now, it suffices to check that (i) $M \in \operatorname{Mod}(T)$ and (ii) $\|A \Rightarrow B\|_{M} \neq 1$.

Ad (i): Let $C \Rightarrow D \in T$. We need to show $\| C \Rightarrow$ $D \|_{M}=1$, i.e. $S(C, M)^{*} \leq S(D, M)$ which is equivalent to $S(C, M)^{*} \otimes D \subseteq M$. Hence, it is sufficient to show that $T \vdash{ }_{\omega} A \Rightarrow S(C, M)^{*} \otimes D$ which is indeed true. In more detail, we have that

$T \vdash{ }_{\omega} A \Rightarrow M$

[see above],

$T \vdash_{\omega} M \Rightarrow S(C, M)^{*} \otimes C$

[instance of $(\mathrm{Ax})]$,

$T \vdash{ }_{\omega} S(C, M)^{*} \otimes C \Rightarrow S(C, M)^{*} \otimes D \quad$ [by (Mul) on $\left.C \Rightarrow D\right]$,

Thus, $T \vdash_{\omega} A \Rightarrow S(C, M)^{*} \otimes D$ follows by (Tra).

Ad (ii): By contradiction, suppose $\|A \Rightarrow B\|_{M}=1$. Since $A \subseteq M$ by definition, we then get $1=\|A \Rightarrow B\|_{M}=$ $S(A, M)^{*} \rightarrow S(B, M)=1 \rightarrow S(B, M)=S(B, M)$, i.e. $B \subseteq$ $M$. Since $T \vdash_{\omega} A \Rightarrow M$, (Pro) would give $T \vdash_{\omega} A \Rightarrow B$, a contradiction.

Theorem 7 (completeness) Let $T$ be a set of FAIs. Then

$$
T \vdash{ }_{\omega} A \Rightarrow B \quad \text { iff } \quad\|A \Rightarrow B\|_{T}=1 .
$$

Proof. The " $\Rightarrow$ "-part follows from Theorem 5. For the " $\Leftarrow$ "-part, observe that due to Lemma 6 , if $T \forall_{\omega} A \Rightarrow B$, then there is $M \in \operatorname{Mod}(T)$ such that $\|A \Rightarrow B\|_{M} \neq 1$, i.e. $\|A \Rightarrow B\|_{T} \neq 1$, which finishes the proof.

In addition to the previous characterization of fully entailed FAIs, we can consider a degree $|\cdots|_{T}^{\omega}$ of $\omega$ provability of FAIs and show completeness in graded style $\left(|\cdots|_{T}^{\omega}\right.$ agrees with $\left.\|\cdots\|_{T}\right)$. In more detail, for a set $T$ of
FAIs and for $A \Rightarrow B$ we define a degree $|A \Rightarrow B|_{T} \in L$ to which $A \Rightarrow B$ is provable from $T$ by

$$
|A \Rightarrow B|_{T}^{\omega}=\bigvee\left\{c \in L \mid T \vdash_{\omega} A \Rightarrow c \otimes B\right\} .
$$

Now, we have the following

Theorem 8 (graded completeness) For each set $T$ of FAIs and each $A \Rightarrow B$, we have $|A \Rightarrow B|_{T}^{\omega}=\|A \Rightarrow B\|_{T}$.

Proof. The claim follows by Theorem 7 and using $\| A \Rightarrow$ $B \|_{T}=\bigvee\left\{c \in L\|\| A \Rightarrow c \otimes B \|_{T}=1\right\}$, see [3].

We can go even farther: we can consider inference from fuzzy sets of FAIs and still be able to have a graded completeness for any $\mathbf{L}$. Due to the lack of space, this and related results are postponed to the full version of the paper.

Acknowledgement Supported by grant No. 1ET101370417 of GA AV ČR, by grant No. 201/05/0079 of the Czech Science Foundation, and by institutional support, research plan MSM 6198959214.

\section{References}

[1] Bělohlávek R.: Fuzzy Relational Systems: Foundations and Principles. Kluwer, Academic/Plenum Publishers, New York, 2002.

[2] Bělohlávek R., Chlupová M., Vychodil V.: Implications from data with fuzzy attributes. In: Proc. AISTA 2004, 5 pages, ISBN 2-9599776-8-8.

[3] Bělohlávek R., Vychodil V.: Fuzzy attribute logic: syntactic entailment and completeness. In: Proc. JCIS 2005, pp. 78-81, ISBN 0-9707890-3-3.

[4] Bělohlávek R., Vychodil V.: Fuzzy attribute logic: attribute implications, their validity, entailment, and non-redundant basis. In: Proc. IFSA 2005, vol. I, pp. 622-627, ISBN 7-302-11377-7.

[5] Bělohlávek R., Vychodil V.: Axiomatizations of fuzzy attribute logic. In: Proc. IICAI 2005, pp. 2178-2193, ISBN 0-9727412-1-6.

[6] Bělohlávek R., Vychodil V.: Attribute implications in a fuzzy setting. In: Missaoui R., Schmid J. (Eds.): ICFCA 2006, LNAI 3874, pp. 45-60, 2006.

[7] Grimaldi R.,P.: Discrete and Combinatorial Mathematics, An Appl. Introd., Pearson Education, 2004.

[8] Hájek P.: Metamathematics of Fuzzy Logic. Kluwer, Dordrecht, 1998.

[9] Hájek P.: On very true. Fuzzy Sets and Systems 124(2001), 329-333.

[10] Maier D.: The Theory of Relational Databases. Computer Science Press, Rockville, 1983.

[11] Takeuti G., Titani S.: Globalization of intuitionistic set theory. Annals of Pure and Applied Logic 33(1987), 195-211.

[12] Wechler W.: Universal Algebra for Computer Scientists. Springer-Verlag, Berlin Heidelberg, 1992. 